\title{
Morphological Analysis of Corneal Opacity in Shiba Dog with GM1 Gangliosidosis
}

\author{
Aya NAGAYASU ${ }^{1)}$, Taito NAKAMURA ${ }^{1)}$,Osamu YAMATO ${ }^{2)}$, Keiko TSUZUKI ${ }^{3)}$, Yoshinao HOSAKA ${ }^{1)}$, \\ ${\text { Hiromi } \text { UEDA }^{1)} \text {, Prasarn TANGKAWATTANA }}^{4)}$ and Kazushige TAKEHANA ${ }^{1)}$
}

${ }^{1)}$ Department of Veterinary Anatomy, ${ }^{3)}$ Veterinary Teaching Hospital, Rakuno Gakuen University, 582 Bunkyodai-Midorimachi, Ebetsu, Hokkaido 069-8501, ${ }^{2)}$ Laboratory of Clinical Pathology, Department of Veterinary Clinical Sciences, Faculty of Agriculture, Kagoshima University, 1-2121-24 Kohrimoto, Kagoshima 890-0065, Japan and ${ }^{4)}$ Department of Veterinary Anatomy, Faculty of Veterinary Medicine Khon Kean University, Khon Kean 40002, Thailand

(Received 10 March 2008/Accepted 27 April 2008)

ABSTRACT. GM1 gangliosidosis is one of the inherited metabolic lysosomal storage disorders characterized by neurological symptoms caused by $\beta$-galactosidase deficiency and consequent accumulation of GM1 ganglioside in neuronal cells. Shiba dogs affected with GM1 gangliosidosis have been found to suffer from corneal opacity. In our morphological analysis, keratocyte enlargement was induced by abnormal intracellular accumulation of neutral carbohydrates, resulting in the loss of normal arrangement of collagen fibrils in the opaque cornea was found to be associated with the disorder. We therefore conclude that corneal opacity in this Shiba dog with GM1 gangliosidosis may be caused by neutral carbohydrate accumulation in lysosomes, swelling and dysfunction of keratocytes, and subsequent irregular arrangement of collagen fibrils in the corneal proper substance.

KEY WORDS: corneal opacity, GM1 gangliosidosis, keratocyte, Shiba dog.

In normal cells, the lysosome contains more than 60 kinds of acid hydrolases responsible for catabolism of various complex carbohydrates and complex lipids. Deficiency in these enzymes results in the excessive storage of their substrates, leading to the so-called lysosomal storage disorder characterized by progressive cell damage and organ dysfunction. The disorder has been described in various species; including dogs, cats, cattle, goats, sheep, mice, and humans $[1-7,11,20,29,30]$. Depending on the type of excessively accumulated substrates, the disorder can be subclassified as sphingolipidosis, mucopolysaccharidosis, glycoprotein degrading disorders, and mucolipidosis among others. GM1 gangliosidosis is a type of sphingolipidosis typcally characterized by excessive GM1 ganglioside accumulation in the lysosome of various cells and tissues. This is due to the lack of $\beta$-galactosidase responsible for catabolizing GM1 ganglioside to GM2 ganglioside in the sphingolipid metabolic pathway $[15,22]$. Deficiency in $\beta$-galactosidase arises from mutations of the GLB1 gene [28]. The excessive storage of GM1 ganglioside causes retention of complex carbohydrates with a $\beta$-galactose terminal leading to the systemic pathological conditions of GM1 gangliosidosis, particularly in cells and tissues of the central nervous system. These complex carbohydrates in cells and tissues can be specifically detected by established methods for carbohydrate histochemical analyses described in various reports [13, 23-27]. Typical GM1 gangliosidosis manifests cellular swelling and distension, functional impairment, and neurological signs including tremor and ataxic paralysis. In Shiba dogs, the onset of the disorder occurs at 5-6 months of age. Besides these clinical manifestations, affected dogs

\footnotetext{
* Correspondence to: Ueda, H., Rakuno Gakuen University, Hokkaido 069-8501, Japan.

e-mail: hueda@rakuno.ac.jp
}

at about 10 months also develop corneal opacity, which is rarely observed in other breeds, and eventually die at about 15 months [29]. To further clarify the pathogenesis of the disorder, this work focused on the histological and carbohydrate histochemical analyses of the opaque cornea of a Shiba dog with GM1 gangliosidosis.

\section{MATERIALS AND METHODS}

The study protocol for the use of experimental animals was approved by the Ethics Committee of Hokkaido University, Sapporo, Japan (the previous institution of Osamu Yamato). In this experiment, a 13-month-old Shiba dog with GM1 gangliosidosis showing the symptom of corneal opacity was used [29]. The affected dog was born in 2002 and belonged to the family pedigree of Shiba dogs [30]. The dog was diagnosed based on clinical signs and the results of enzyme [21] and DNA mutation [28] assays. In addition, an adult beagle with normal ocular and physical health was used as a control. The eyes of these dogs were examined clinically by slit-lamp examination and direct ophthalmoscopy to determine the patterns of corneal opacity. The dogs were euthanized by an appropriate dose of thiopental sodium (Ravonal, Tanabe Seiyaku Co., Ltd., Osaka, Japan) and corneal samples were immediately collected. Affected corneal samples were selected in the area showing opacity.

At room temperature, the corneal samples $(1 \times 1 \times 1 \mathrm{~mm}$ in dimension) were fixed in $3.0 \%$ glutaraldehyde in $0.1 \mathrm{M}$ phosphate buffer ( $\mathrm{pH} \mathrm{7.4)}$ for $2 \mathrm{hr}$, post-fixed in $1.0 \%$ osmium tetroxide in $0.1 \mathrm{M}$ phosphate buffer for $1 \mathrm{hr}$, washed with distilled water, dehydrated in a graded ethanol series, and embedded in Quetol 812 (Nissin EM, Tokyo, Japan). Semithin sections were stained with $2 \%$ toluidine blue and examined by light microscopy. Histological 
examinations of all corneal layers and their keratocyte population were performed and the number of keratocytes per $0.01 \mathrm{~mm}^{2}$ was calculated to determine keratocyte density (cells $/ 0.01 \mathrm{~mm}^{2}$ ). Ultrathin sections were stained with $1.0 \%$ uranyl acetate and $1.0 \%$ lead citrate, and examined using a transmission electron microscope (TEM; JEM-1220; JEOL, Tokyo, Japan) at an accelerating voltage of $80 \mathrm{kV}$. From the randomly selected TEM photographs of each sample, the structure of keratocytes, the ratio of intracellular vesicles to the whole cytoplasmic area of each keratocyte, and 900 fibrils for the measurement of collagen fibril parameters were examined. The average intracellular vesicle ratio and collagen fibril diameter were calculated. For carbohydrate histochemistry, semithin and ultrathin sections were stained with a solution of periodic acid (PA) and diamine with high (HID) or low iron concentration (LID) at room temperature for $60 \mathrm{~min}$, and then washed in distilled water for 10-15 min. At room temperature, the sections were incubated in $0.2 \%$ thiocarbohydrazide (TCH, Eastman Kodak, U.S.A.) in $20 \%$ acetic acid for $60 \mathrm{~min}$, rinsed in distilled water for 10 $15 \mathrm{~min}$, immersed in a $0.5 \%$ aqueous solution of silver protein (SP, Merck, Germany) in a dark box for $60 \mathrm{~min}$, and washed in distilled water for 10-15 min. The sections were developed at $20^{\circ} \mathrm{C}$ in the dark box for $12-15 \mathrm{~min}$, washed in distilled water for 10-15 min, treated with physical developer (PD) for 5-10 seconds, and then rinsed in distilled water for 10-15 min (PA-TCH-SP-PD for neutral carbohydrates; HID-TCH-SP-PD for sulfated carbohydrates; LIDTCH-SP-PD for carboxylated carbohydrates). Staining with uranyl acetate and lead citrate was not performed. Then, intracellular neutral and acidic carbohydrates of keratocytes were observed by light and transmission electron microscopies.

Student's $t$-test was used for data analysis. $\mathrm{P}<0.05$ was considered statistically significant.

\section{RESULTS}

Clinical observation revealed irregularly circular or ringshaped patterns of corneal opacity and different opacity shapes on the sides in the Shiba dog with GM1 gangliosidosis (Fig. 1a, b). By slit-lamp examination, opacity was observed only in the anterior corneal proper substance (Fig. 1c, d).

Morphologically, the anterior and posterior epithelial layers of the cornea in the affected and control dogs showed no difference, except for the morphological changes observed only in the corneal proper substance. The arrangement of collagen fibrils in the corneal proper substance of the affected dog was less organized (Fig. 2a) than that of the control dog, which showed a more regular pattern (Fig. 2b). In the control dog, the keratocytes were morphologically flat and slender with strongly stained cytoplasm. These cells were located between regularly arranged collagen fibril lamellae (Fig. 2d). In the affected dog, swollen keratocytes with vesicular cytoplasm were found between disarranged fibril lamellae (Fig. 2c). The cytoplasmic vesicles were membranous with various sizes and contained fine electrondense particles (Fig. 3a). Lamellated structures were also found in some of these vesicles (Fig. 3b). Since some differ-
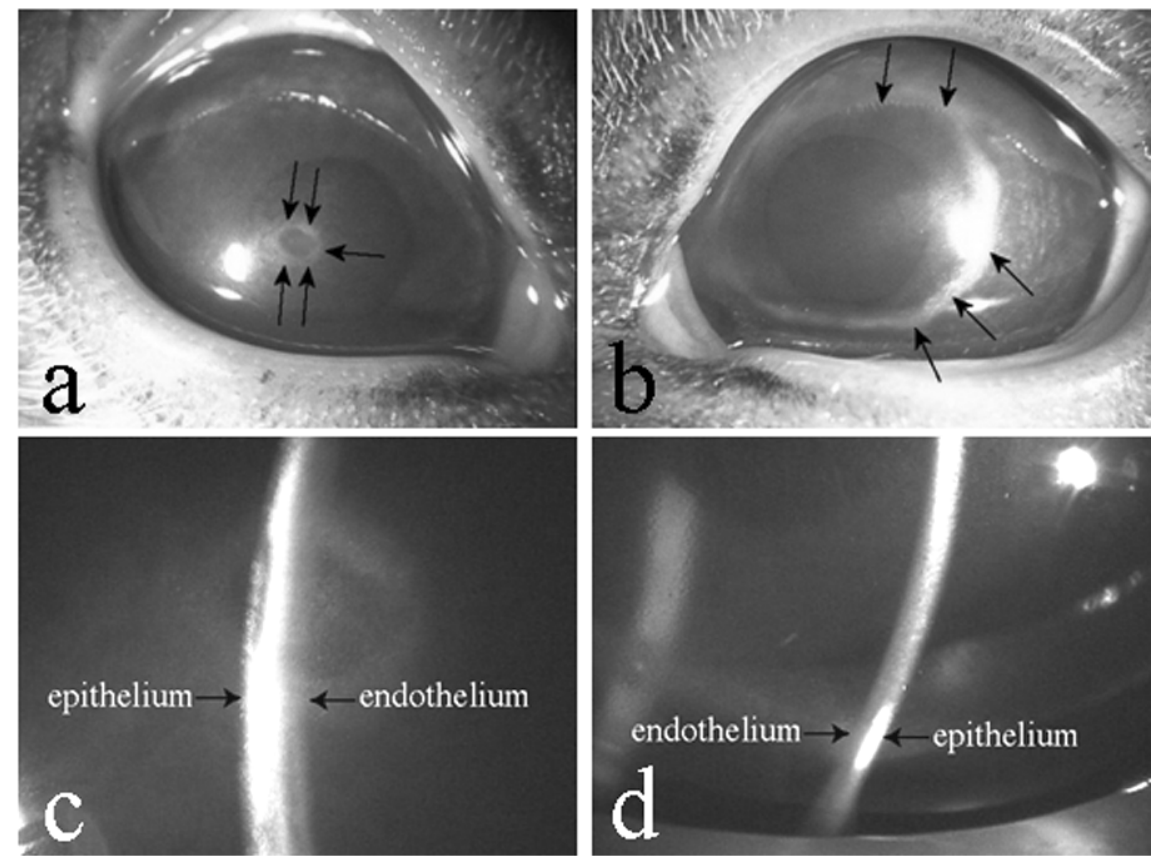

Fig. 1. Clinical appearance of the right $(a, c)$ and left $(b, d)$ eyes of the affected dog. Corneal opacity is observed in both eyes $(a, b)$. Slit-lamp examination reveals opacity only in the anterior region of the corneal stroma $(\mathrm{c}, \mathrm{d})$. 

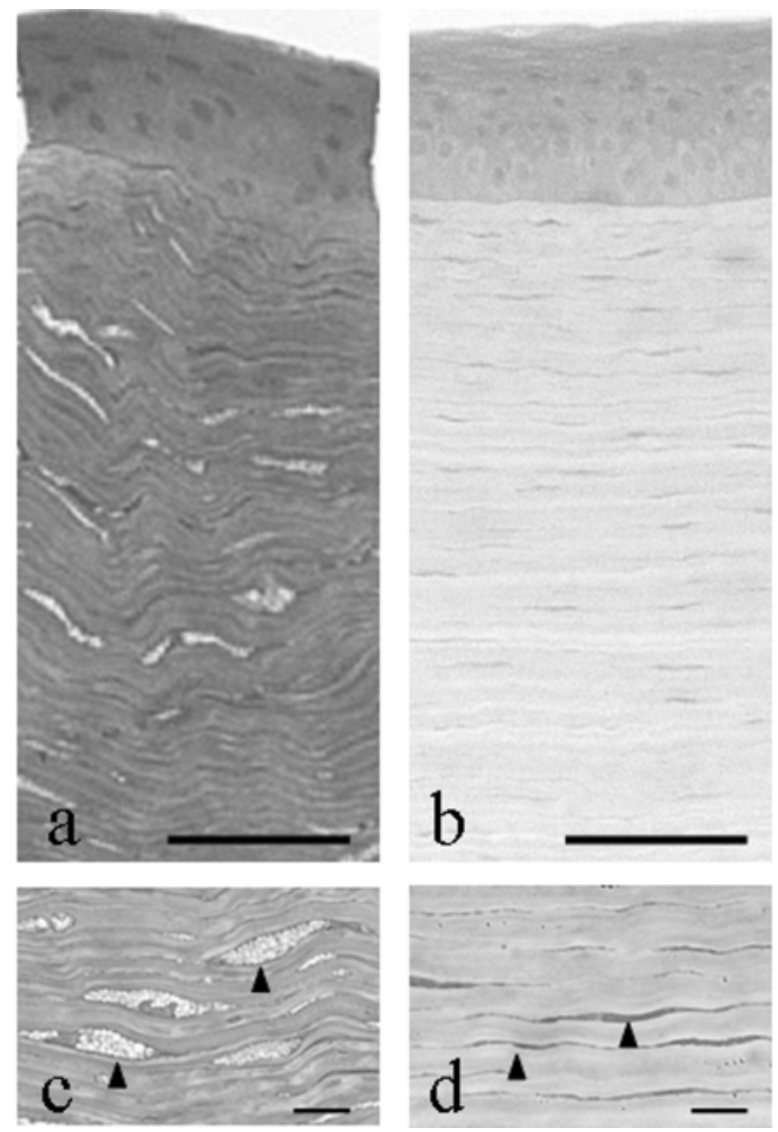

Fig. 2. Light micrographs showing the corneal stroma. In the affected $\operatorname{dog}(\mathrm{a}, \mathrm{c})$, the collagen fibers are irregularly arranged and the keratocytes (arrowheads) are swollen and filled with vesicles. In the control dog, regular arrangement of the collagen fibers intersperses with flat and slender keratocytes (arrowheads) are observed only in the control dog $(b, d)$. Scale bars $=$ $50 \mu \mathrm{m}(\mathrm{a}, \mathrm{b})$ and $5 \mu \mathrm{m}(\mathrm{c}, \mathrm{d})$. ences in carbohydrate contents of these vesicles were assumed, three different histochemical stainings were carried out. A positive reaction was found only for neutral carbohydrates, but not for acidic carbohydrates (Fig. 4).

Keratocyte density in three different regions (upper, middle, and lower) of the cornea was found to vary only in the affected dog. The keratocyte densities of the affected dog vs the control dog in the upper, middle, and lower regions were $24.8 \pm 4.6$ vs $11.8 \pm 1.5,15.8 \pm 1.5$ vs $10.9 \pm 1.5$, and $17.4 \pm$ 4.7 vs $10.2 \pm 0.5$ cells $/ 0.01 \mathrm{~mm}^{2}$, respectively. Keratocyte densities in all the regions of the affected dog were significantly different from those of the control $\operatorname{dog}(\mathrm{P}<0.05)$, particularly in the upper region (Fig. 5). The average diameter of a collagen fibril in all the regions of the affected dog (26.3 $\pm 2.8 \mathrm{~nm}$ ) was significantly smaller than that of the control $\operatorname{dog}(28.3 \pm 2.8 \mathrm{~nm})(\mathrm{P}<0.05$; Fig. 6$)$.

\section{DISCUSSION}

In the present study, corneal opacity was found in the Shiba dog and the affected area was limited to the anterior corneal proper substance. However, since other inherited metabolic diseases, such as Hurler syndrome (a subtype of mucopolysaccharidosis) and Fabry disease (a type of sphingolipidosis), also produce different opacity patterns [9, $10,14,16,19]$, the shape of the opaque lesion might not be type-specific. Variations in corneal opacity patterns might be due to site of localization and intensity of the affected area.

Histologically, the canine cornea is composed of four consecutive layers: anterior epithelium, proper substance, Descemet's membrane, and posterior epithelium [18]. Among these layers, the proper substance constitutes about $90 \%$ of the entire cornea in terms of thickness. The corneal proper substance of the control dog was formed by stacks of regularly arranged collagenous lamellae interspersed with many flat and elongated keratocytes. It has been shown that corneal transparency is generally maintained by the regular interfibrillar spacing of collagen fibrils with characteristic
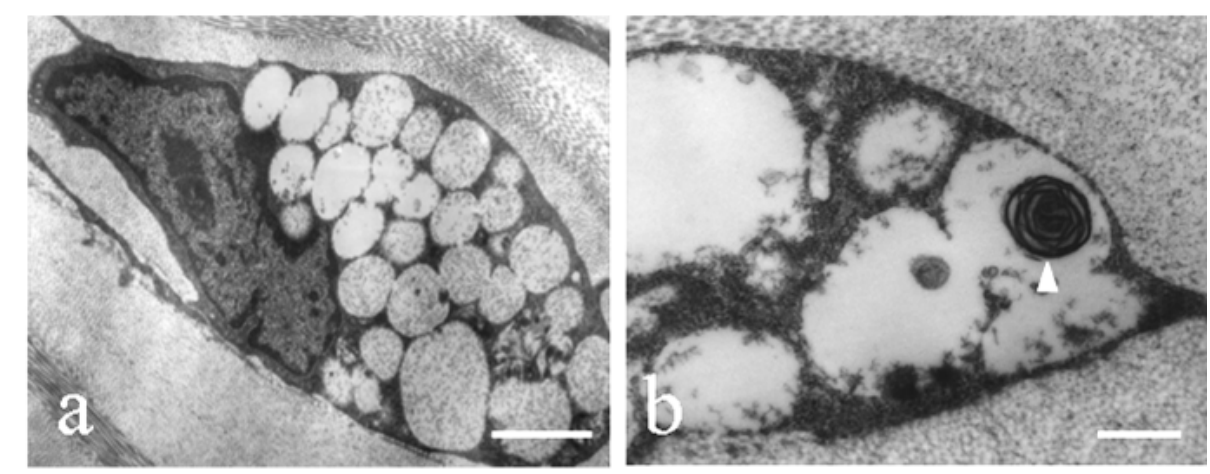

Fig. 3. Transmission electron micrographs showing a keratocyte in the affected dog. The cell is filled with various sizes of membranous and electron lucent vesicles. Some vesicles contain fine electrondense particles (a). A lamellated structure in the electron lucent vesicles of a keratocyte is observed (b, arrowhead). Scale bars $=2 \mu \mathrm{m}$ (a) and $500 \mathrm{~nm}$ (b). 

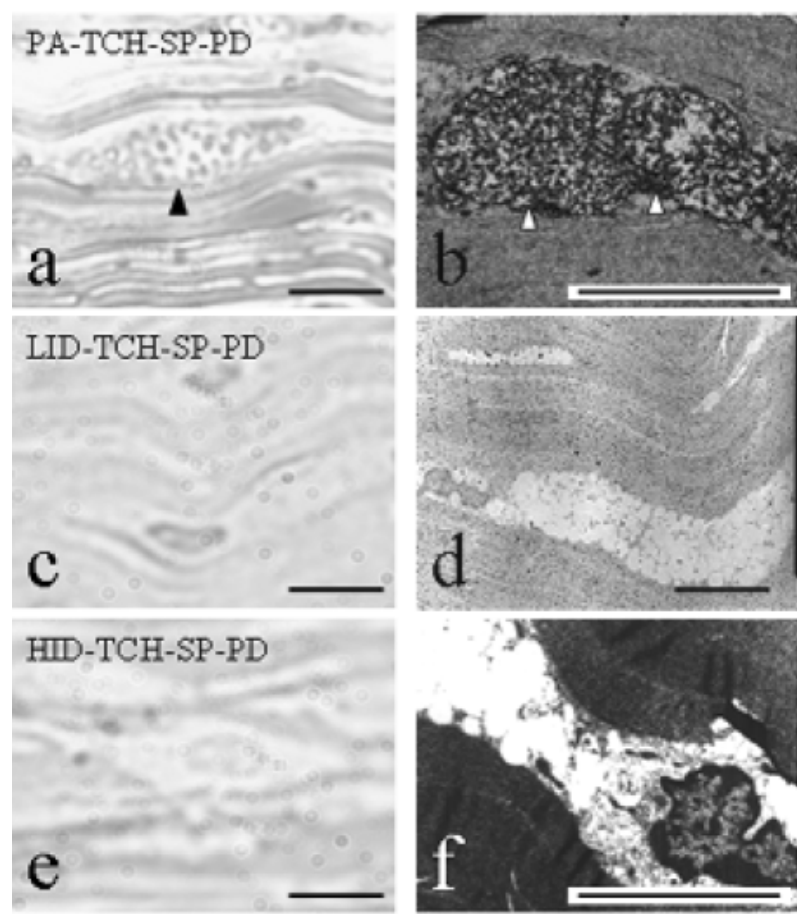

Fig. 4. Light (a, c, e) and transmission electron (b, d, f) micrographs showing histochemical reactions of three different carbohydrates contained in the vesicles of keratocytes; PA-TCH-SPPD (a, b), LID-TCH-SP-PD (c, d), and HID-TCH-SP-PD (e, f). Positive reaction is observed in the vesicles ( $\mathrm{a}, \mathrm{b}$ : arrowheads). Scale bar $=5 \mu \mathrm{m}$.

uniform diameter [17]. Therefore, abnormalities within the proper substance might contribute to the reduction of transparency. Specifically, the causes of an adverse condition are the following: edema, abnormalities in diameter and arrangement of collagen fibrils similarly found in scar and fibrotic tissues, abnormal accumulation of macromolecules (proteins, glycosaminoglycans, lipids, etc.) similarly found

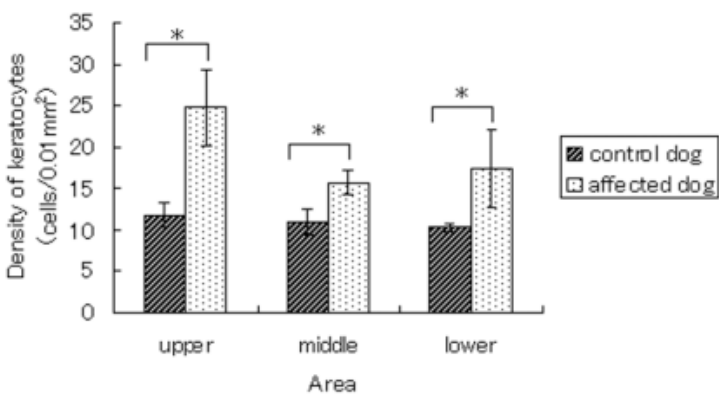

Fig. 5. Keratocyte density in the three different regions of the corneal stroma of the affected and control dogs. The density shows slight variations in the control dog. The highest density is observed in the upper stromal region of the affected dog. The difference in keratocyte density is significant in all pairs of comparison $(*, p<0.05)$.

in corneal dystrophy, and abnormal light reflectivity of keratocytes [12]. In the present study, the presence of a corneal opaque ring restricted in the anterior corneal proper substance correlated well with high keratocyte density. Thus, this correlation indicates that abnormalities in keratocyte density implicate the formation of this condition.

GM1 gangliosidosis is characterized by accumulation of complex carbohydrates in neuronal cells together with cellular swelling and distention [29]. It is well known that keratocytes in other inherited metabolic diseases such as mucopolysaccharidosis accumulate acidic carbohydrates in the form of glycosaminoglycans in their lysosomes [10, 19]. However, the keratocytes of the Shiba dog in the present study accumulated only neutral carbohydrates in their vesicles. The lamellated structure observed in some intracellular vesicles could indicate an aged lysosome or residual body [8]. The increase in the number of vesicles can induce swelling and disturb the cellular function involved in fibril production as well as the arrangement of surrounding collagen fibrils. Normally, the collagen fibril population of the
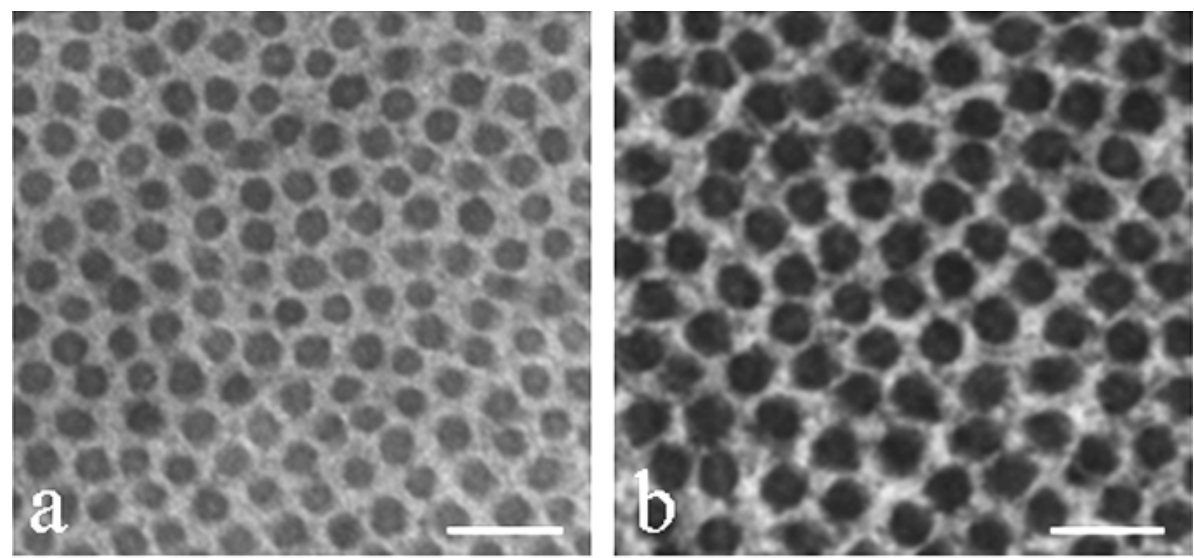

Fig. 6. Transmission electron micrographs showing collagen fibril diameter of the affected (a) and control (b) dogs. The collagen fibrils of the affected dog are thinner than those of the control dog. Scale bar $=100 \mathrm{~nm}$. 
corneal proper substance is synthesized by keratocytes. However, since the fibrils observed in the affected dog were thinner than those observed in the control dog, we assume that differences in the metabolic function of keratocytes affect normal fibril production. Moreover, these attenuated fibrils are more sensitive to the compressive effects of swollen keratocytes and are thus easily disarranged.

Corneal opacity is observed in human GM1 gangliosidosis, particularly in the infantile type of this disease [15, 22]. The cause of the corneal opacity in human GM1 gangliosidosis might be the same as that in canine GM1 gangliosidosis because the canine disease closely resembles the human disease genetically, clinically, biochemically and pathologically $[22,29]$.

In conclusion, the corneal opacity in the Shiba dog with GM1 gangliosidosis in the present study is likely to be the sequel of abnormal accumulation of neutral carbohydrates in the lysosomes of corneal keratocytes. This accumulation induces keratocyte dysfunction and swelling finally resulting in the irregular arrangement of collagen fibrils in the corneal proper substance.

\section{REFERENCES}

1. Ahern-Rindell, A. J., Prieur, D. J., Murnane, R. D., Raghavan, S. S., Daniel, P. F., McCluer, R. H., Walkley, S. U. and Parish, S. M. 1988. Inherited lysosomal storage disease associated with deficiencies of beta-galactosidase and alpha-neuraminidase in sheep. Am. J. Med. Genet. 31: 39-56.

2. Alroy, J., Orgad, U., DeGasperi, R., Richard, R., Warren, C. D., Knowles, K., Thalhammer, J. G. and Raghavan, S. S. 1992. Canine GM1-gangliosidosis. A clinical, morphologic, histochemical, and biochemical comparison of two different models. Am. J. Pathol. 140: 675-689.

3. Alroy, J., Orgad, U., Ucci, A. A., Schelling, S. H., Schunk, K. L., Warren, C. D., Raghavan, S. S. and Kolodny, E. H. 1985. Neurovisceral and skeletal GM1-gangliosidosis in dogs with beta-galactosidase deficiency. Science 229: 470-472.

4. Baker, H. J. and Lindsey, J. R. 1974. Animal model: feline GM1 gangliosidosis. Am. J. Pathol. 74: 649-652.

5. Cork, L. C., Munnell, J. F. and Lorenz, M. D. 1978. The pathology of feline GM2 gangliosidosis. Am. J. Pathol. 90: 723-734.

6. De Maria, R., Divari, S., Bo, S., Sonnio, S., Lotti, D., Capucchio, M. T. and Castagnaro, M. 1998. Beta-galactosidase deficiency in a Korat cat: a new form of feline GM1gangliosidosis. Acta Neuropathol. 96: 307-314.

7. Driemeier, D., Colodel, E. M., Gimeno, E. J. and Barros, S. S. 2000. Lysosomal storage disease caused by Sida carpinifolia poisoning in goats. Vet. Pathol. 37: 153-159.

8. Fawcett, D. W. and Raviola, E. 1994. THE CELL. pp. 1-56. In: A Textbook of Histology, 12th ed., Chapman \& Hall, New York.

9. Hirano, K., Murata, K., Miyagawa, A., Terasaki, H., Saigusa, J., Nagasaka, T. and Kobayashi, M. 2001. Histopathologic findings of cornea verticillata in a woman heterozygous for Fabry's disease. Cornea 20: 233-236.

10. Huang, Y., Bron, A. J., Meek, K. M., Vellodi, A. and McDonald, B. 1996. Ultrastructural study of the cornea in a bone marrow-transplanted Hurler syndrome patient. Exp. Eye
Res. 62: 377-387.

11. Jolly, R. D. and Walkley, S. U. 1997. Lysosomal storage diseases of animals: an essay in comparative pathology. Vet. Pathol. 34: 527-548.

12. Moller-Pedersen, T. 2004. Keratocyte reflectivity and corneal haze. Exp. Eye Res. 78: 553-560.

13. Nakamura, M., Kitamura, H. and Yamada, K. 1985. A sensitive method for the histochemical demonstration of vicinal diols of carbohydrates. Histochem. J. 17: 477-485.

14. Nguyen, T. T., Gin, T., Nicholls, K., Low, M., Galanos, J. and Crawford, A. 2005. Ophthalmological manifestations of Fabry disease: a survey of patients at the Royal Melbourne Fabry Disease Treatment Centre. Clin. Experiment. Ophthalmol. 33: 164-168.

15. O'Brien, J. S. 1989. $\beta$-galactosidase deficiency (GM1 gangliosidosis, galactosialidosis and Morquio syndrome type B); Ganglioside sialidase deficiency (mucolipidosis). pp. 17971806. In: The Metabolic Basis of Inherited Disease, 6th ed. (Scriver, C.R., Beaudet, A. L., Sly, W. S. et al. eds.), McGrawHill, New York.

16. Ries, M., Gupta, S., Moore, D. F., Sachdev, V., Quirk, J. M., Murray, G. J., Rosing, D. R., Robinson, C., Schaefer, E., Gal, A., Dambrosia, J. M., Garman, S. C., Brady, R. O. and Schiffmann, R. 2005. Pediatric Fabry disease. Pediatrics 115: e344355.

17. Samuelson, D. A. 1991. Ophthalmic embryology and anatomy. pp. 3-123. In: Veterinary Ophthalmology, 2nd ed. (Gellat, K.N. ed.), Lea and Febiger, Philadelphia.

18. Shively, J. N. and Epling, G. P. 1970. Fine structure of the canine eye: cornea. Am. J. Vet. Res. 31: 713-722.

19. Shull, R. M., Kakkis, E. D., McEntee, M. F., Kania, S. A., Jonas, A. J. and Neufeld, E. F. 1994. Enzyme replacement in a canine model of Hurler syndrome. Proc. Natl. Acad. Sci. U S A 91: 12937-12941.

20. Skelly, B. J., Jeffrey, M., Franklin, R. J. and Winchester, B. G. 1995. A new form of ovine GM1-gangliosidosis. Acta Neuropathol. 89: 374-379.

21. Suzuki, K. 1978. Enzymic diagnosis of sphingolipidoses. Methods Enzymol. 50: 456-488.

22. Suzuki, K., Oshima, A. and Nanba, E. 2001. $\beta$-galactosidase deficiency ( $\beta$-galacatosidosis): GM1gangliosidosis and Morquio B disease. pp. 3775-3809. In: The Metabolic and Molecular Bases of Inherited Disease, 8th ed. (Scriver, C.R., Beaudet, A. L., Sly, W. S. et al. eds.), McGrawHill, New York.

23. Takehana, K., EERDUNCHAOLUO, Ueda, H., Kobayashi, A., Iwasa, K. and Sou, K. 2000. A histochemical study of the camel (Camelus bactrianus) duodenal glands. J. Vet. Med. Sci. 62: $449-452$.

24. Takehana, K., Abe, M., Iwasa, K., Hiraga, T. and Miyata, H. 1991. Carbohydrate histochemistry of bovine duodenal glands. J. Vet. Med. Sci. 53: 699-706.

25. Ueda, H. and Fujimori, O. 1998. Effects of a triple enzyme digestion method on a diamine reaction for glycosaminoglycans of the rat aorta in electron microscopy. J. Vet. Med. Sci. 60: $1169-1174$.

26. Ueda, H. and Fujimori, O. 1995. Carbohydrate cytochemistry of the endothelium lining the splenic blood vessels in the rat. Okajimas Folia Anat. Jpn. 72: 109-117.

27. Ueda, H., Fujimori, O. and Abe, M. 1996. Histochemical analysis of acidic glycoconjugates in the endothelium lining the splenic blood vessels in the rat. Arch. Histol. Cytol. 59: 389-397.

28. Yamato, O., Endoh, D., Kobayashi, A., Masuoka, Y., Yone- 
mura, M., Hatakeyama, A., Satoh, H., Tajima, M., Yamasaki, M. and Maede, Y. 2002. A novel mutation in the gene for canine acid beta-galactosidase that causes GM1-gangliosidosis in Shiba dogs. J. Inherit. Metab. Dis. 25: 525-526.

29. Yamato, O., Masuoka, Y., Yonemura, M., Hatakeyama, A., Satoh, H., Kobayashi, A., Nakayama, M., Asano, T., Shoda, T., Yamasaki, M., Ochiai, K., Umemura, T. and Maede, Y. 2003.
Clinical and clinico-pathologic characteristics of Shiba dogs with a deficiency of lysosomal acid beta-galactosidase: a canine model of human GM1 gangliosidosis. J. Vet. Med. Sci. 65: 213-217.

30. Yamato, O., Ochiai, K., Masuoka, Y., Hayashida, E., Tajima, M., Omae, S., Iijima, M., Umemura, T. and Maede, Y. 2000. GM1 gangliosidosis in shiba dogs. Vet. Rec. 146: 493-496. 